\title{
ARTICLE
}

\section{Tuning thermal conductivity in molybdenum disulfide by electrochemical intercalation}

Gaohua Zhu ${ }^{1, \star}$, Jun Liü ${ }^{2,3, \star}$, Qiye Zheng ${ }^{2}$, Ruigang Zhang ${ }^{1}$, Dongyao Li $^{2}$, Debasish Banerjee ${ }^{1}$ \& David G. Cahill ${ }^{2}$

Thermal conductivity of two-dimensional (2D) materials is of interest for energy storage, nanoelectronics and optoelectronics. Here, we report that the thermal conductivity of molybdenum disulfide can be modified by electrochemical intercalation. We observe distinct behaviour for thin films with vertically aligned basal planes and natural bulk crystals with basal planes aligned parallel to the surface. The thermal conductivity is measured as a function of the degree of lithiation, using time-domain thermoreflectance. The change of thermal conductivity correlates with the lithiation-induced structural and compositional disorder. We further show that the ratio of the in-plane to through-plane thermal conductivity of bulk crystal is enhanced by the disorder. These results suggest that stacking disorder and mixture of phases is an effective mechanism to modify the anisotropic thermal conductivity of 2D materials.

\footnotetext{
${ }^{1}$ Materials Research Department, Toyota Research Institute of North America, Ann Arbor, Michigan 48105, USA. ${ }^{2}$ Department of Materials Science and Engineering, Frederick Seitz Materials Research Laboratory, University of Illinois at Urbana-Champaign, Urbana, Illinois 61801, USA. ${ }^{3}$ Department of Mechanical and Aerospace Engineering, North Carolina State University, Raleigh, North Carolina 27695, USA. * These authors contributed equally to this work. Correspondence and requests for materials should be addressed to G.Z. (email: gaohua.zhu@toyota.com) to or J.L. (email: jun_liu@ncsu.edu) or to D.G.C. (email: d-cahill@illinois.edu).
} 
T wo-dimensional (2D) layer-structured materials consist of atomic layers with strong intra-layer covalent bonding stacked together by weak van der Waals bonds. Transition metal dichalcogenides, an important class of $2 \mathrm{D}$ materials, have attracted extensive research interest recently due to their unique electronic and chemical properties 1 . In particular, molybdenum disulfide $\left(\mathrm{MoS}_{2}\right)$ has been extensively studied for potential applications in nanoelectronics, optoelectronics and flexible electronic devices ${ }^{2-5}$. Although the thermal conductivity of single, few-layer and bulk $\mathrm{MoS}_{2}$ has been reported recently, the effects of structural and compositional disorder on the anisotropic thermal conductivity of layered materials, which usually occurs during crystal growth, fabrication and applications (for example, in energy storage, thermoelectrics and nanoelectronics), have not yet been systematically characterized $^{6-8}$.

Guest ions can be intercalated into the van der Waals gaps in $\mathrm{MoS}_{2}$. Intercalation causes changes in the electronic structure, and optical and electrical properties ${ }^{9}$. Intercalation can also induce structural and compositional disorder, including variations in layer spacing, interaction strengths between adjacent layers and phase transitions $s^{10-12}$. By monitoring the potential during electrochemical intercalation, we can control the amount of intercalated ions. Therefore, intercalation provides an effective way to systematically vary the structural and compositional disorder of many 2D materials, and enables investigations of how disorder affects their thermal conductivity.

To understand how thermal conductivity of highly anisotropic materials can be affected by disorder, here we study the thermal conductivity of both pristine and lithium ion-intercalated bulk and thin-film $\mathrm{MoS}_{2}$. In the bulk sample, the $\mathrm{MoS}_{2}$ basal planes are oriented parallel to the surface; whereas, in the thin-film sample, the $\mathrm{MoS}_{2}$ basal planes are vertically aligned. The thermal conductivity of $\mathrm{Li}_{\mathrm{x}} \mathrm{MoS}_{2}$ samples with different degrees $(x)$ of electrochemical interaction of lithium ions were measured by time-domain thermoreflectance (TDTR). We show that lithium ion intercalation has drastically different effects on thermal transport in these different forms of $\mathrm{MoS}_{2}$ due to the differences in crystalline orientation and initial structural disorder. Our most striking observation is that the thermal anisotropy ratio in bulk $\mathrm{Li}_{x} \mathrm{MoS}_{2}$ crystals increases from $52(x=0)$ to 110 $(x=0.34)$ as a result of lithiation-induced stacking disorder and phase transitions. The thermal anisotropy ratio is the ratio of in-plane to through-plane thermal conductivity, an important material parameter in thermal management. The increase in thermal anisotropy with increasing disorder is counter-intuitive: previous studies show that structural disorder typically decreases the thermal anisotropy ratio ${ }^{13,14}$. Our analysis suggest that the enhanced thermal anisotropy ratio in $\mathrm{Li}_{x} \mathrm{MoS}_{2}$ bulk crystal is likely due to the combination of phonon-focusing effects and pronounced differences in the inplane and through-plane length scale of the lithiation-induced disorder.

\section{Results}

Sample preparation. The $\mathrm{MoS}_{2}$ thin-film samples with vertically aligned basal planes were grown by rapid sulfurization of a Mo thin film ${ }^{15}$. Bulk samples of $\mathrm{MoS}_{2}$ were obtained by mechanical exfoliation of bulk $\mathrm{MoS}_{2}$ crystals (SPI Supplies). The cross-sectional transmission electron microscopy (TEM) image in Fig. 1a reveals that the film thickness is $\approx 200 \mathrm{~nm}$ after chemical-vapour deposition (CVD) growth and the $\mathrm{MoS}_{2}$ atomic layers are predominantly aligned perpendicular to the substrate with the edges of the $\mathrm{MoS}_{2}$ layers exposed to the surface. A typical plan-view TEM image of the vertically aligned $\mathrm{MoS}_{2}$ thin film is shown in Fig. 1b. The thin film is polycrystalline with randomly oriented strip-like or columnar grains. The cross-sectional area of those columnar grains is $\sim 10 \mathrm{~nm}$ wide and several tens of nanometres long. Bulk $\mathrm{MoS}_{2}$ samples, with typical thickness of $10-20 \mu \mathrm{m}$, were prepared by standard Scotch tape assisted mechanical exfoliation. A plan-view TEM image of the bulk $\mathrm{MoS}_{2}$ sample is shown in Fig. 1c, where the high-quality atomic plane of $\mathrm{MoS}_{2}$ can be seen.

Further structural analysis of bulk and thin-film $\mathrm{MoS}_{2}$ samples before lithiation were carried out by X-ray diffraction and Raman spectroscopy. In X-ray diffraction, bulk samples show a strong (002) peak located at $2 \theta=14.4^{\circ}$, and (004), (006) and (008) peaks with decaying magnitudes, indicating that the basal planes of the bulk sample are parallel to the surface. The thin-film samples have two peaks located at $32.8^{\circ}$ and $58.5^{\circ}$, which are due to the diffraction from (100) and (110) planes of individual columnar grains, consistent with our conclusion from the TEM images that the basal planes are vertically aligned (see details in Fig. 5). In contrast to the bulk sample, (002) peak is not observed in the X-ray diffraction spectrum of the thin-film sample because the normal to the (002) planes is parallel to the substrate surface.

Figure 2a,b present the Raman spectra of the bulk and thinfilm $\mathrm{MoS}_{2}$ samples. To probe the low-frequency interlayer $E_{2 g}^{2}$ phonon mode, we used three reflective volume Bragg grating filters (BragGrate notch filters) in combination with a single-pass monochromator to access frequency shifts as small as $\sim 10 \mathrm{~cm}^{-1}$. The $E_{1 g}$ mode $\left(286 \mathrm{~cm}^{-1}\right)$ is observed only in the thin-film samples while the $E_{2 g}^{2}$ mode $\left(32 \mathrm{~cm}^{-1}\right)$ is only present in the bulk samples, as expected. According to the Raman selection rules, the $E_{1 g}$ mode is forbidden in backscattering experiment on the basal plane of bulk $\mathrm{MoS}_{2}$ (refs 16,17). However, when the incident light scatters on the surface of edge-terminated $\mathrm{MoS}_{2}$, the corresponding scattering Raman tensor undergoes a rotation transformation, leading to a nonzero differential scattering cross-section and hence the $E_{1 g}$ mode can be observed. The observation of the $E_{1 g}$ mode in thin-film samples therefore indicates that the basal planes of $\mathrm{MoS}_{2}$ are vertically aligned, consistent with the TEM and X-ray diffraction data. The absence of the $E_{2 g}^{2}$ mode (which is not forbidden by selection rules) in thin-film samples is probably due to the randomly oriented columnar grains and stacking disorder in CVD-grown samples. In addition, although both $A_{1 g}$ at $383 \mathrm{~cm}^{-1}$
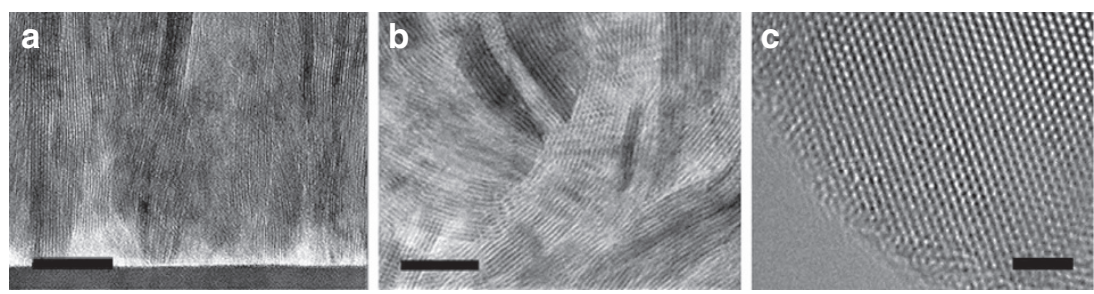

Figure 1 | TEM images of $\mathbf{M o S}_{\mathbf{2}}$ bulk crystals and thin films. (a) Cross-sectional TEM image of the MoS 2 thin film with vertically aligned basal plane. Scale bar, $20 \mathrm{~nm}$. (b) Plan-view TEM image of the $\mathrm{MoS}_{2}$ thin film. Scale bar, $10 \mathrm{~nm}$. (c) Plan-view TEM image of the bulk MoS 2 crystal. Scale bar, $2 \mathrm{~nm}$. 

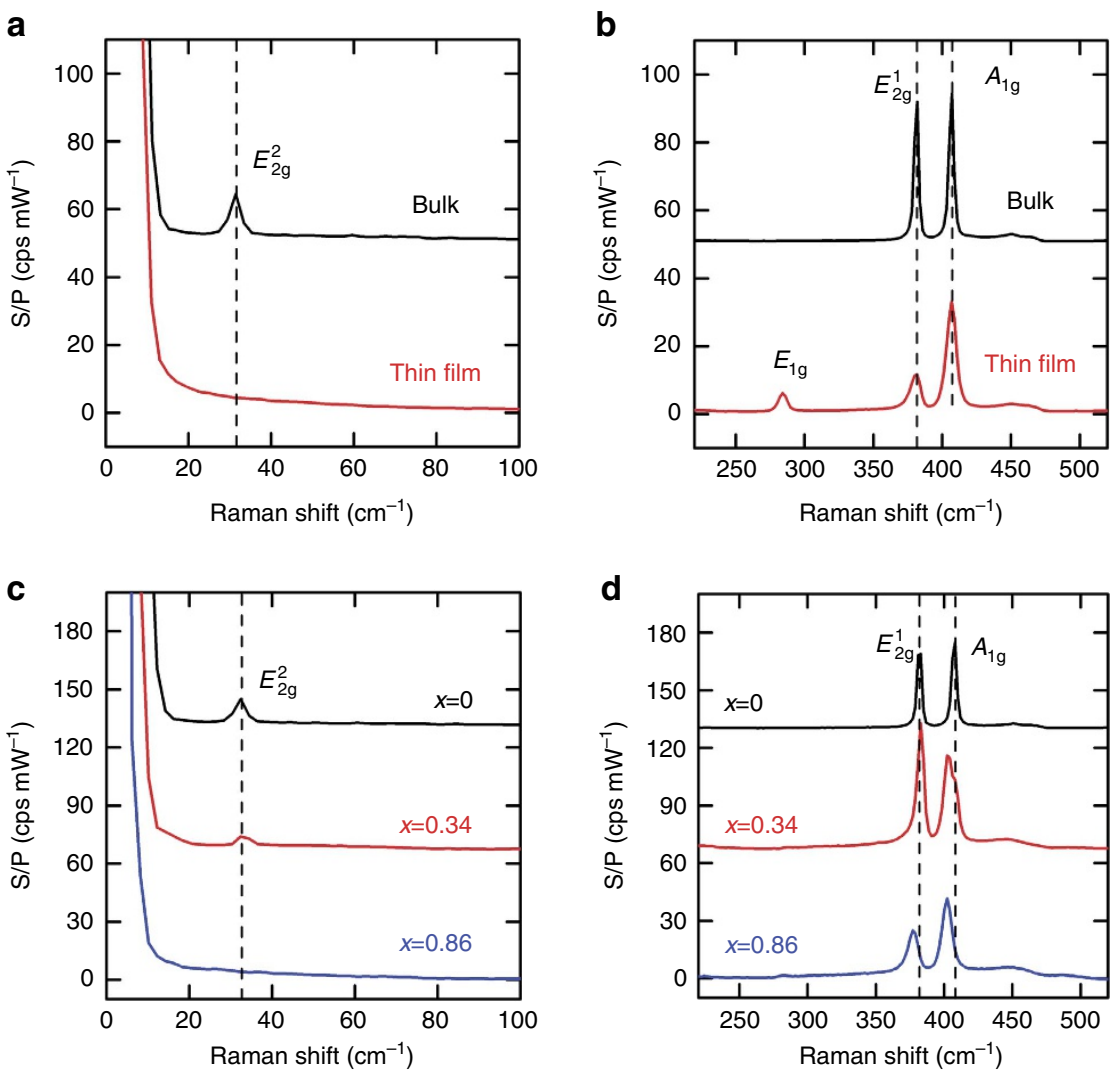

Figure 2 | Raman spectra for bulk and thin-film $\mathbf{M o S}_{\mathbf{2}}$ samples before lithiation. Spectra obtained at (a) low frequencies and (b) high frequencies. The $y$-axis is the signal intensity normalized by laser power (S/P) in the unit of counts per second per milliwatt (cps mW $\mathrm{m}^{-1}$ ). The bulk spectrum is shifted up by $50 \mathrm{cps} \mathrm{mW}^{-1}$. Raman spectra for bulk $\mathrm{Li}_{x} \mathrm{MoS}_{2}$ samples at different degrees of lithiation $(x=0,0.34$ and 0.68$)$ at (c) low frequencies, (d) high frequencies. The $x=0.34$ and $x=0$ spectra are shifted up by 65 and $130 \mathrm{cps} \mathrm{mW}^{-1}$, respectively.

and $E_{2 g}^{1}$ at $408 \mathrm{~cm}^{-1}$ modes are present in bulk and thin-film $\mathrm{MoS}_{2}$, the peak intensity of the out-of-plane $A_{g}^{1}$ mode is similar to that of the in-plane $E_{2 g}^{1}$ mode in the bulk sample and $\sim 3$ times that of the $E_{2 g}^{1}$ mode in the thin-film sample under the same measurement condition. Such preferred excitation of an out-ofplane mode is also consistent with the vertical-aligned crystal texture of the thin-film sample considering the polarization dependence of the Raman scattering cross-section ${ }^{15}$.

We carried out electrochemical intercalation of lithium ions in both bulk and thin-film $\mathrm{MoS}_{2}$ to study how lithiation affects the thermal conductivity differently in $\mathrm{MoS}_{2}$ samples with different orientations. Lithium ion intercalation of thin-film $\mathrm{MoS}_{2}$ samples was performed through a galvanostatic discharge process in a glass vial inside a glovebox; electrochemical intercalation of bulk $\mathrm{MoS}_{2}$ samples as performed using a coin cell battery setup ${ }^{18}$. In both cases, $\mathrm{MoS}_{2}$ samples were used as the working electrode, and the lithium foil was used as the counter and reference electrode.

The discharge curves for thin-film and bulk $\mathrm{MoS}_{2}$ samples are shown in Fig. 3. On lithium ion intercalation, a well-defined plateau is observed at potentials between 1.1 and $1.2 \mathrm{~V}$, as the host lattice of $\mathrm{MoS}_{2}$ undergoes a phase transition from $2 \mathrm{H}$ to $1 \mathrm{~T}$ phase ${ }^{19}$. The voltage dip at the initial stage of the discharge curve for bulk $\mathrm{MoS}_{2}$ sample is caused by the mass transport limitation of lithium ions. The voltage gradually recovers when the lithium ion transport is facilitated by defects formed during intercalation. The voltage dip was not observed in the discharge process of thin-film $\mathrm{MoS}_{2}$, which can be attributed to the high density of the edge sites in edgeterminated thin-film samples.

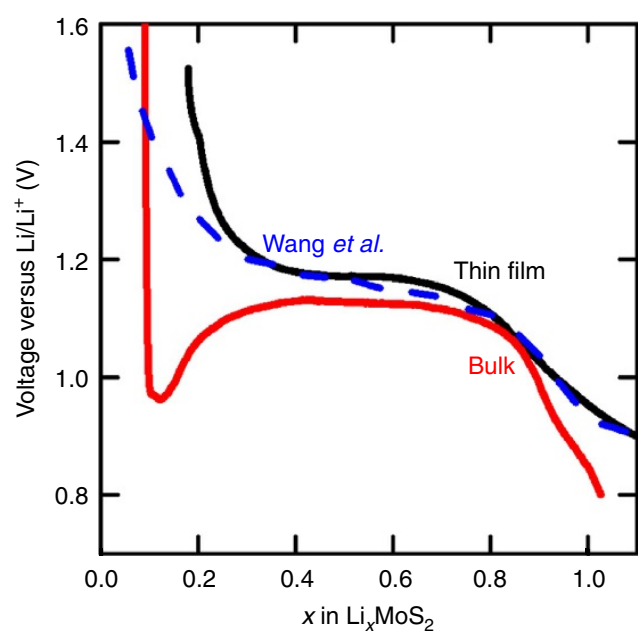

Figure 3 | Initial discharge curves for $\mathbf{M o S}_{\mathbf{2}}$ samples. Thin-film (black) and bulk (red) $\mathrm{MoS}_{2}$ samples, compared with data by Wang et al. ${ }^{55}$ (dashed line) for thin-film $\mathrm{Li}_{x} \mathrm{MoS}_{2}$.

We describe the lithium ion intercalation process in the range of $1.1-3.0 \mathrm{~V}$ as reaction (1):

$$
\begin{aligned}
& \mathrm{MoS}_{2}+x \mathrm{Li}^{+}+x \mathrm{e}^{-} \rightarrow \mathrm{Li}_{x} \mathrm{MoS}_{2} \\
& \quad\left(\sim 1.1 \mathrm{~V} \text { versus } \mathrm{Li} / \mathrm{Li}^{+}, 0 \leq x \leq 1\right)
\end{aligned}
$$

We calculate the average lithium composition from the electronic charge transferred to $\mathrm{MoS}_{2}$, based on the theoretical specific 
charge capacity for full intercalation of $167 \mathrm{~mA} \mathrm{hg}^{-1}$ (ref. 19). Hence, by controlling the duration of the galvanostatic discharge process as shown in Fig. 3, vertically aligned $\mathrm{Li}_{x} \mathrm{MoS}_{2}$ thin-film $(x=33$ at\%, 46 at\%, 68 at $\%$ and 100 at\%) and lithiated bulk samples (20 at $\%, 40$ at $\%, 60$ at $\%, 80$ at $\%$ and 100 at $\%$ ) with various lithiation content $(x)$ were prepared.

Since the charge transfer during electrochemical intercalation can have a contribution from complicated side reactions, the actual degree of lithiation $(x)$ for each sample might be different from the calculated values. To determine the actual $x$ in bulk $\mathrm{MoS}_{2}$ samples, inductively coupled plasma mass spectroscopy measurements were performed on one fully lithiated bulk sample. The mass ratio of $\mathrm{Li}, \mathrm{Mo}$ and $\mathrm{S}$ atoms is calculated by measuring the element masses in the bulk $\mathrm{Li}_{x} \mathrm{MoS}_{2}$ sample with metal coating (total mass $\approx 1 \mathrm{mg}$ ). The measured $x$ is 86 at $\%$ suggesting reasonable agreement between calculated and measured lithium contents for the lithiation process. The actual $x$ in other bulk $\mathrm{Li}_{x} \mathrm{MoS}_{2}$ samples is then scaled by the same factor of 0.86 .

Thermal conductivity. $\mathrm{MoS}_{2}$ samples (schematics shown in Fig. $4 \mathrm{a}$ ) were characterized by TDTR to determine the change in thermal conductivity caused by lithium ion intercalation. The TDTR data of both thin-film and bulk $\mathrm{Li}_{x} \mathrm{MoS}_{2}$ samples are presented as a function of $x$ in Fig. $4 \mathrm{~b}$. The through-plane thermal conductivity of thin-film $\mathrm{Li}_{x} \mathrm{MoS}_{2}$ decreases monotonically from $\approx 3.4 \mathrm{~W} \mathrm{~m}^{-1} \mathrm{~K}^{-1}(x=0)$ to $\approx 1.7 \mathrm{~W} \mathrm{~m}^{-1} \mathrm{~K}^{-1}(x=1)$ with increasing lithium content. The through-plane thermal conductivity of bulk $\mathrm{Li}_{x} \mathrm{MoS}_{2}$ decreases first from $\approx 2.0 \mathrm{~W} \mathrm{~m}{ }^{-1} \mathrm{~K}^{-1}$ $(x=0)$ to $\approx 0.4 \mathrm{~W} \mathrm{~m}^{-1} \mathrm{~K}^{-1}(x=0.34)$ and then increases to $\approx 1.6 \mathrm{~W} \mathrm{~m}^{-1} \mathrm{~K}^{-1}(x=0.86)$. The in-plane thermal conductivity follows a similar trend, which decreases from $\approx 105 \mathrm{~W} \mathrm{~m}^{-1} \mathrm{~K}^{-1}$ $(x=0)$ to $\approx 45 \mathrm{~W} \mathrm{~m}^{-1} \mathrm{~K}^{-1}(x=0.34)$ and then increases to $\approx 80 \mathrm{~W} \mathrm{~m}^{-1} \mathrm{~K}^{-1}(x=0.86)$.

To understand such a drastic decrease of thermal conductivity, we calculated the minimum thermal conductivity $\Lambda_{\min }$ of $\mathrm{Li}_{x} \mathrm{MoS}_{2}$ using a simplified model by Cahill et al. ${ }^{20}$ at the high-temperature limit. The minimum thermal conductivity is $\Lambda_{\min }=\left(\frac{\pi}{48}\right)^{\frac{1}{3}} k_{B} n^{2 / 3}\left(v_{L}+2 v_{t}\right)$, where $k_{B}$ is the Boltzmann constant, $n$ is the atomic density (atoms $\mathrm{cm}^{-3}$ ), $v_{L}$ is the longitudinal speed of sound, and $v_{t}$ is the transverse speed of sound ${ }^{21}$. Atomic densities of $\mathrm{Mo}$ and $\mathrm{S}$ atoms in $\mathrm{Li}_{x} \mathrm{MoS}_{2}$ thin films was measured by Rutherford backscattering spectrometry; $v_{L}$ is measured by picosecond acoustics; $v_{t}$ is measured by detecting surface acoustic waves using a phase-shift mask ${ }^{22}$. We accounted for the change of atomic density and longitudinal speed of sound due to lithiation. More details can be found in the 'Methods' section and the Supplementary Methods.

In thin-film $\mathrm{Li}_{x} \mathrm{MoS}_{2}$ samples, the polycrystalline structure with randomly oriented columnar grains is transverse isotropic so that this conventional minimum thermal conductivity model can be applied. In the bulk $\mathrm{Li}_{x} \mathrm{MoS}_{2}$ samples, however, the strongly anisotropic structure introduces a significant phonon-focusing effect $^{23}$, which suppresses the through-plane average group velocity due to the relatively high in-plane group velocity. We adopted the modified minimum thermal conductivity model recently proposed by Zhen et al. ${ }^{41}$ (equation $\mathrm{S}(7)$ in their Supplementary Material) and followed their procedure to calculate the minimum thermal conductivity of bulk $\mathrm{Li}_{x} \mathrm{MoS}_{2}$ (see details in Supplementary Note 1 with parameters shown in Supplementary Table 1).

If the measured lowest through-plane thermal conductivity of bulk or thin-film $\mathrm{Li}_{x} \mathrm{MoS}_{2}$ agrees with the predicted minimum thermal conductivity, the phonons are glass-like lattice vibrations in a disordered crystal at this composition. The calculated minimum thermal conductivity of bulk and thin-film $\mathrm{Li}_{x} \mathrm{MoS}_{2}$ is
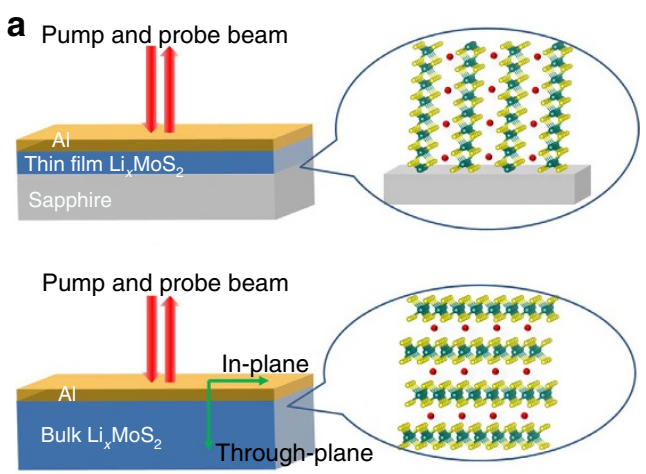

b

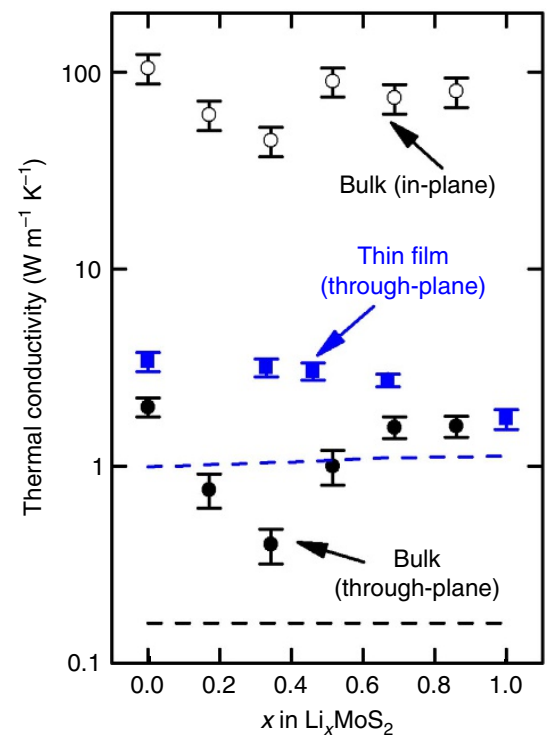

Figure 4 | Thermal conductivity measurement of $\mathrm{Li}_{x} \mathbf{M o S}_{\mathbf{2}}$ samples.

(a) Schematics of bulk and thin-film $\mathrm{Li}_{x} \mathrm{MoS}_{2}$ samples for TDTR measurements. (b) Thermal conductivity of $\mathrm{Li}_{x} \mathrm{MoS}_{2}$ samples with different degrees of lithiation $x$. Blue squares: through-plane thermal conductivity of thin-film $\mathrm{MoS}_{2}$; black squares: through-plane thermal conductivity of bulk $\mathrm{MoS}_{2}$; black open squares: in-plane thermal conductivity of bulk MoS 2 . The minimum thermal conductivity for bulk and thin-film samples are plotted as black and blue dashed lines, respectively. The total uncertainties of the measured thermal conductivity are calculated by taking into account the systematic errors that propagate from uncertainties in the film thickness, laser spot size, and thermal properties of the transducer film and substrate.

plotted in Fig. 4b as dashed lines. Both measured through-plane thermal conductivity of bulk and thin-film $\mathrm{Li}_{x} \mathrm{MoS}_{2}$ is higher than the predicted minimum thermal conductivity, which suggests that a significant fraction of the phonons in these samples are propagating modes.

Interlayer distance characterization. Large expansion in layer spacing along the $c$-axis is often observed after intercalation of large-diameter molecules or ions ${ }^{11,24}$. We collected X-ray diffraction spectra for bulk $\mathrm{Li}_{x} \mathrm{MoS}_{2}$ samples with different amount of lithium ion intercalation $(0 \leq x \leq 0.86)$ to determine the change in layer spacing due to lithium ion intercalation. Samples were placed inside an air-tight sample holder with a beryllium $(\mathrm{Be})$ window in an argon-filled glovebox before they were transferred out for X-ray diffraction characterization. Figure 5b shows that the (002) peak of the pristine $\mathrm{MoS}_{2}$ is located at $14.40^{\circ}$, and the peak position downshifts to $14.28^{\circ}$ for $\mathrm{Li}_{0.86} \mathrm{MoS}_{2}$. The corresponding layer spacing is 6.16 and $6.19 \AA$ 

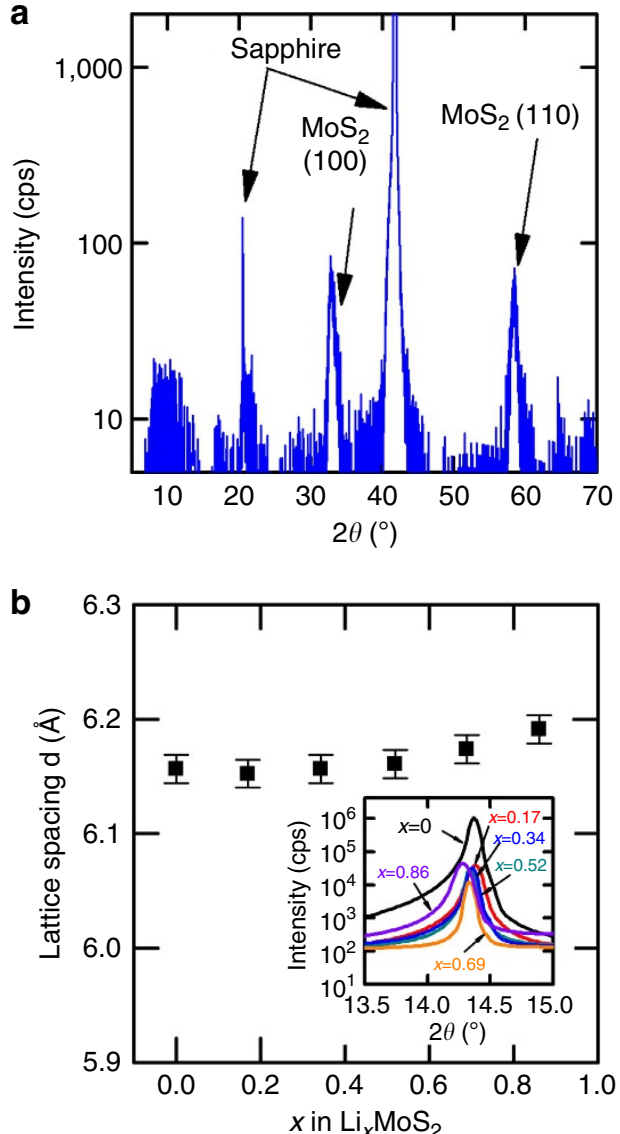

Figure 5 | X-ray diffraction characterization of $\mathrm{Li}_{\mathbf{x}} \mathbf{M o S}_{\mathbf{2}}$ samples.

(a) X-ray diffraction spectra for $\mathrm{MoS}_{2}$ thin-film samples on sapphire substrate. The two diffraction peaks coincide with the standard X-ray diffraction powder patterns of $\mathrm{MoS}_{2}$ (100) and (110). Therefore, the dominant lattice orientation in the $\mathrm{MoS}_{2}$ thin-film samples are (100) and (110). The diffraction peak at $32.8^{\circ}$ and $58.5^{\circ}$ corresponds to a lattice constant of 2.73 and $1.58 \AA$, respectively. (b) Lattice spacing $d$ between $\mathrm{MoS}_{2}$ layers in bulk $\mathrm{MoS}_{2}$ samples. The error bars are calculated by taking into account the experimental errors and the systematic errors that propagate from uncertainties in the fitting of $X$-ray diffraction spectra. The inset plot is the change of $\mathrm{MoS}_{2}(002)$ peak position with $x$ in $\mathrm{Li}_{x} \mathrm{MoS}_{2}$.

for pristine $\mathrm{MoS}_{2}$ and $\mathrm{Li}_{0.86} \mathrm{MoS}_{2}$, respectively, a $0.5 \%$ change in lattice constant. We estimate the uncertainty of the lattice spacing measurement as $\approx 0.6 \%$. The weak dependence of lattice constant on lithium content is consistent with the fact the effective ionic radius of lithium ion ${ }^{25}(76 \mathrm{pm})$ is slightly smaller than the octahedral site in the van der Waals gap of in $\mathrm{MoS}_{2}$. If we assume an effective ionic radius of $\mathrm{S}^{2-}$ as $1.84 \AA$, and close packing of $\mathrm{S}^{2-}$ atoms, the radius of the octahedral site is $76 \mathrm{pm}$ (ref. 25). Our measurement on the layer spacing of $\mathrm{Li}_{0.86} \mathrm{MoS}_{2}$ agrees with recent observations reporting on minimal change in interlayer distance of $\mathrm{LiMoS}_{2}$ (refs 26,27).

Elastic constants. We further measured elastic properties using pump-probe techniques to help understand the thermal conductivity change in our samples. Polycrystalline $\mathrm{MoS}_{2}$ thin films with vertically aligned basal planes are transverse isotropic, which has five effective independent averaged elastic constants: $C_{11}^{\prime}, C_{12}^{\prime}$, $C_{13}^{\prime}, C_{33}^{\prime}$ and $C_{44}^{\prime}$. Figure 6 plots the effective $C_{33}^{\prime}$ and $C_{44}^{\prime}$ elastic constants of thin-film $\mathrm{Li}_{x} \mathrm{MoS}_{2}$. The elastic constant $C_{33}^{\prime}$ of $\mathrm{Li}_{x} \mathrm{MoS}_{2}$ thin film decreases from $147 \mathrm{GPa}(x=0)$ to $121 \mathrm{GPa}$

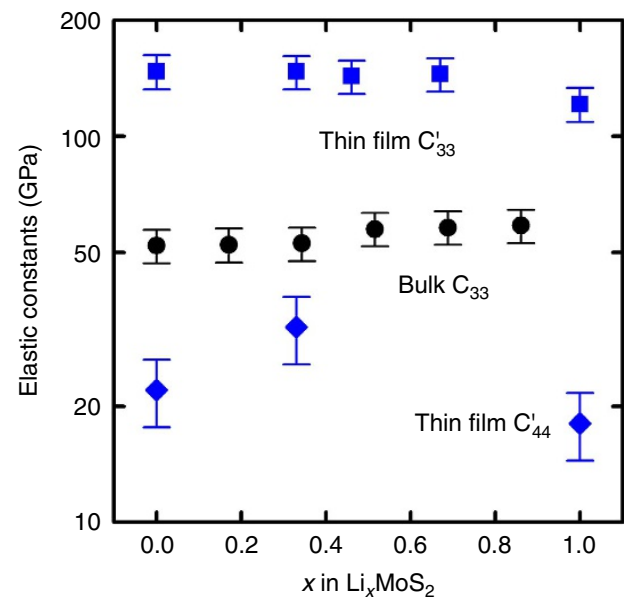

Figure 6 | Effective elastic constant of thin-film $\mathrm{Li}_{x} \mathrm{MoS}_{\mathbf{2}}$ with different degrees of lithiation. $C_{33}^{\prime}$ (square) decreases from $147 \mathrm{GPa}(x=0)$ to $121 \mathrm{GPa}(x=1) . C_{44}^{\prime}$ (diamond) increases from $22 \mathrm{GPa}(x=0)$ to $32 \mathrm{GPa}$ $(x=0.34)$ and then decreases to $18 \mathrm{GPa}(x=1)$. As comparison, the $C_{33}$ of bulk $\mathrm{Li}_{x} \mathrm{MoS}_{2}$ samples (circle) are also plotted. $\mathrm{C}_{33}$ gradually changes from $52 \mathrm{GPa}(x=0)$ to $58 \mathrm{GPa}(x=0.86)$ with a transition point at $x \approx 0.34$. The error bars are calculated by taking into account the experimental errors and the systematic errors that propagate from uncertainties in the Al film thickness, $\mathrm{Li}_{x} \mathrm{MoS}_{2}$ film density and the input elastic constants.

$(x=1)$. Even though the density of $\mathrm{Li}_{x} \mathrm{MoS}_{2}$ thin films increases by $11 \%$ from $x=0$ to $x=1$, the decrease of longitudinal speed of sound from $5,720 \mathrm{~ms}^{-1}$ to $4,930 \mathrm{~ms}^{-1}$ (measured by picosecond acoustics) dominates the decrease of $C_{33}^{\prime}$. The elastic constant $C_{44}^{\prime}$ increases from $22 \mathrm{GPa}(x=0)$ to $32 \mathrm{GPa}(x=0.34)$ and then decreases to $18 \mathrm{GPa}(x=1)$. We do not yet understand the trend of the elastic constants of thin-film $\mathrm{Li}_{x} \mathrm{MoS}_{2}$ changing with $x$. One possible reason is the combined effect of increasing binding energy due to the intercalation of lithium ions and increasing structural and compositional disorder (for example, point defects and mixture of phases). More measurement details are described in the 'Methods' section and Supplementary Methods.

The elastic constants of bulk $\mathrm{Li}_{x} \mathrm{MoS}_{2}$ samples $C_{33}$ are also plotted in Fig. 6. $C_{33}$ gradually changes from $52 \mathrm{GPa}(x=0)$ to $58 \mathrm{GPa}(x=0.86)$ with a transition point at $x \approx 0.34$, which suggests a phase transition in bulk $\mathrm{Li}_{x} \mathrm{MoS}_{2}$.

Raman spectroscopy. We used Raman spectroscopy to further characterize lithiation-induced structural and compositional disorder of bulk $\mathrm{Li}_{x} \mathrm{MoS}_{2}$ to gain more insights about other phonon scattering mechanisms that could lead to the thermal conductivity change. All bulk $\mathrm{Li}_{x} \mathrm{MoS}_{2}$ samples except pristine $\mathrm{MoS}_{2}$ were loaded inside the glovebox into a home-made air-free sample holder sealed by O-ring and screw-on connectors and measured through the glass window of the holder. As shown in Fig. 2c,d, the intensity of the low-frequency peak at $\sim 32 \mathrm{~cm}^{-1}$ corresponding to the $E_{2 g}^{2}$ shear mode in $2 \mathrm{H}-\mathrm{MoS}_{2}$ decreases as the degree of lithiation $(x)$ increases. The decreasing peak intensity of $E_{2 g}^{2}$ mode is attributed to the increasing stacking disorder resulting from the lithium ion intercalation. In the $2 \mathrm{H}$ to $1 \mathrm{~T}$ phase transition, the stacking of Mo atom planes changes from ABA (two molecular layers per unit cell) to AA (one molecular plane per unit cell) ${ }^{28,29}$. As the atomic structure of the $\mathrm{MoS}_{2}$ layer changes from prismatic $a B a(2 \mathrm{H})$ structure to octahedral $a B c(1 \mathrm{~T})$ (upper letters correspond to Mo planes, lowercase letters correspond to the $S$ planes) ${ }^{30}$, the high-frequency $E_{2 g}^{1}$ and $A_{1 g}$ modes redshift from 383 to $377 \mathrm{~cm}^{-1}$ and 408 to $402 \mathrm{~cm}^{-1}$, respectively, as the 
lithium content increases from $x=0$ to $x=0.86$. The observed redshift is mainly attributed to the intra-layer shift of the $S$ atom during the $2 \mathrm{H}$ to $1 \mathrm{~T}$ phase change ${ }^{31}$.

\section{Discussion}

Lithium ion intercalation into van der Waals gap could change the thermal transport of $\mathrm{MoS}_{2}$ due to several distinct mechanisms. First, the 2D layer spacing might change as a result of lithiation ${ }^{10,11,19}$. In addition, a recent theoretical work found that intercalated lithium ions enhances the binding energy through orbital hybridizations between cations (lithium ions) and anions $\left(\mathrm{MoS}_{2}\right)$ (ref. 32). Second, the intercalated lithium donates electrons to $\mathrm{MoS}_{2}$, which changes the oxidation states of Mo, and hence the electronic properties of $\mathrm{MoS}_{2}$ (refs 33,34). Finally, intercalation drives a phase transition from the semiconductor $2 \mathrm{H}$ phase to the metallic $1 \mathrm{~T}$ phase ${ }^{19}$.

The increase of electrical conductivity caused by the phase transition from the semiconductor $2 \mathrm{H}$ to metallic $1 \mathrm{~T}$ phase cannot explain the changes in the in-plane thermal conductivity of bulk $\mathrm{Li}_{x} \mathrm{MoS}_{2}$. A recent study shows the in-plane electrical conductivity of the metallic phase $(1 \mathrm{~T}) \mathrm{LiMoS}_{2}$ is $\approx 300 \mathrm{~S} \mathrm{~cm}^{-1}$, $\sim 500$ times that of the semiconducting phase $(2 \mathrm{H}) \mathrm{MoS}_{2}$ (ref. 35). The corresponding electronic contribution to the total thermal conductivity, predicted by the Wiedemann-Franz law, is only $0.2 \mathrm{~W} \mathrm{~m}^{-1} \mathrm{~K}^{-1}$ in $\mathrm{LiMoS}_{2}$. Such a small increase cannot be the predominant mechanism to account for the in-plane thermal conductivity change in bulk $\mathrm{Li}_{x} \mathrm{MoS}_{2}$ from 45 to $80 \mathrm{~W} \mathrm{~m}^{-1} \mathrm{~K}^{-1}$ for $x=0.34$ to 0.86 .

We applied the Leibfried-Schlomann (LS) equation to evaluate the effect of changes in lattice spacing, elastic constants and mass density on the thermal conductivity of $\mathrm{Li}_{x} \mathrm{MoS}_{2}$. The LS equation only considers changes in three-phonon scattering rates and takes the form $\Lambda=\frac{B \bar{M} \omega_{D}^{3} \delta}{T \gamma^{2}}$, where $\bar{M}$ is the mass of a unit cell, $\delta^{3}$ corresponds to atomic volume, $T$ is temperature, $\gamma$ is the Grüneisen parameter for the relevant direction of heat transfer, $\omega_{D}$ is the Debye frequency for the relevant direction of heat transfer, and $B$ is an amalgam of physical constants ${ }^{36}$. Using the Debye frequency defined as $\omega_{D}=v_{D} k_{D}$, where $k_{D}$ is the Debye cutoff vector, $v_{D}$ is the speed of sound, we can express the thermal conductivity $\Lambda=\frac{B^{\prime} \bar{M}}{T \gamma^{2} \delta^{2}}\left(\frac{C_{i j}}{\rho}\right)^{1.5}$, where $B^{\prime}$ is a constant, $C_{i j}$ is the elastic constant and $\rho$ is the density. Considering a $4 \%$ increase in atomic mass of the unit cell, $11 \%$ increase in density, $18 \%$ decrease in the effective elastic constant $C_{33}^{\prime}$ and $0.5 \%$ increase in lattice spacing for $\mathrm{Li}_{x} \mathrm{MoS}_{2}$ thin films from $x=0$ to 1 , the LS equation predicts a $33 \%$ decrease in the through-plane thermal conductivity, if we assume that the change in Grüneisen parameter is negligible. Since TDTR measurement shows that through-plane thermal conductivity of thin-film $\mathrm{Li}_{x} \mathrm{MoS}_{2}$ decreases continuously from $\approx 3.4$ to $\approx 1.7 \mathrm{~W} \mathrm{~m}^{-1} \mathrm{~K}^{-1}$ from $x=0$ to 1 , we conclude that the softening of the lattice is a significant contribution to the reduction in thermal conductivity in $\mathrm{Li}_{x} \mathrm{MoS}_{2}$ thin films.

Similarly for the bulk samples, if we consider the changes of elastic constants, lattice spacing, density and atomic mass in a unit cell to be $11 \%, 0.5 \%, 3.3 \%, 3.8 \%$, respectively from $x=0$ to $x=0.86$, the through-plane thermal conductivity is estimated to increase by $\approx 14 \%$. Under the same assumptions, the throughplane thermal conductivity of $\mathrm{Li}_{x} \mathrm{MoS}_{2}$ should increase by $\approx 5 \%$ from $x=0$ to $x=0.34$. Such a small change and different trends indicate the lattice expansion and elastic constant change cannot explain the pronounced change in the through-plane thermal conductivity that we observed in bulk $\mathrm{Li}_{x} \mathrm{MoS}_{2}$ samples.

Interestingly, Raman spectroscopy of bulk $\mathrm{Li}_{x} \mathrm{MoS}_{2}$ samples revealed that the $A_{1 g}$ mode peak splits into two peaks at 402 and
$408 \mathrm{~cm}^{-1}$, while the $E_{2 g}^{2}$ peak slightly broadens in the $x=0.34$ sample suggesting the co-existence of $2 \mathrm{H}$ and $1 \mathrm{~T}$ phases. The peak splitting was related to the Davydov pairs of the optical phonon branches due to the splitting of intra-layer modes caused by the interlayer interaction when the lithium content is below $x=0.34$ (ref. 29). We do not yet have a good understanding why the split of $E_{2 g}^{2}$ peak is not clearly observed in the $x=0.34$ sample but it may be related to the intermediate atomic structure in the not-completed phasechange process. Both high- and low-frequency Raman spectra provide evidence of increasing stacking disorder and mixture of phases during lithiation.

As a result of co-existence of two phases and increasing stacking disorder, phonon-boundary scattering increases and thermal conductivity in both through-plane and in-plane directions decrease until the largest degree of disorder is reached at $x \approx 0.34$. As intercalation proceeds, the bulk $\mathrm{MoS}_{2}$ sample becomes increasingly dominated by the $1 \mathrm{~T}$ phase $(0.34<x<0.86)$ and the phonon-boundary scattering rate decreases, and hence the thermal conductivity starts to increase again.

Our most striking observation is that the thermal anisotropy ratio of the bulk $\mathrm{Li}_{x} \mathrm{MoS}_{2}$ increases from $52(x=0)$ to 110 $(x=0.34)$ with increasing disorder. This trend of thermal anisotropy ratio change with respect to the structural defects or disorder is indeed counter-intuitive and beyond any previous works. For example, a recent study of graphene oxide films by Renteria et al. ${ }^{14}$ shows an increase of thermal anisotropy ratio attributed to decreasing disorder after annealing. Luckyanova et al. ${ }^{13}$ found that the thermal anisotropy ratio decreases with interface atomic mixing in superlattices. The thermal anisotropy ratio of a disordered $\mathrm{WSe}_{2}$ crystal, $\approx 30$, is similar to the anisotropy ratio of well-ordered $\mathrm{WSe}_{2}$ crystals ${ }^{37,38}$. Our measurements of lithiated $\mathrm{MoS}_{2}$ bulk crystal demonstrate that lithiation-induced stacking disorder and phase transition can increase the thermal anisotropy ratio.

Phonon-phonon and phonon-boundary scattering are the two possible major scattering mechanisms in bulk $\mathrm{Li}_{x} \mathrm{MoS}_{2}$. As discussed above, we estimated the change of the intrinsic lattice thermal conductivity that is limited by phonon-phonon interactions due to the changes in elastic constants and atomic densities. The predicted change in the intrinsic phonon-phonon scattering rates is small and we therefore exclude changes in phononphonon scattering as the dominant mechanism in lithiated bulk $\mathrm{Li}_{x} \mathrm{MoS}_{2}$. The combination of our Raman spectroscopy data-and the pronounced change in the thermal conductivity of bulk samples-suggest that phonon scattering in bulk $\mathrm{Li}_{x} \mathrm{MoS}_{2}$ is dominated by phonon-boundary scattering due to stacking disorder created by the phase transition.

In highly anisotropic layered materials, boundary-limited phonon mean-free paths in the in-plane and through-plane directions can differ by orders-of-magnitude due to the combination of phonon-focusing effects and divergent length scales of disorder in the in-plane versus through-plane directions. When boundary scattering dominates the phonon mean-free paths, phonon-focusing produces anisotropic phonon mean-free path even in cubic crystals ${ }^{39}$. A recent modelling study ${ }^{40}$ demonstrates that thin films of graphite can maintain their high in-plane thermal conductivity even for film thickness as small as $10 \mathrm{~nm}$. In other words, in highly anisotropic crystals, boundary scattering along the $c$-axis has only a minimal effect on the $a b$-plane thermal conductivity. Further experimental evidence of this effect comes from a recent experimental study ${ }^{41}$ of CVDgrown graphite thin films where the phonon mean-free paths are different by more than an order of magnitude: the in-plane phonon mean-free path is $\approx 300 \mathrm{~nm}$ and the through-plane mean-free path is $<20 \mathrm{~nm}$. 
Although a sophisticated phonon transport model is needed to rigorously analyse how different phonon branches are affected by boundaries and other forms of disorder, we can estimate how the boundary-limited phonon mean-free paths in the $c$-axis $L_{c}$ and in the ab-plane $L_{a b}$ change with $x$ by comparing our measured thermal conductivities with previous studies. We compare the measured in-plane thermal conductivities of bulk $\mathrm{Li}_{x} \mathrm{MoS}_{2}$ with the thermal conductivity of single-layer $\mathrm{MoS}_{2}$ predicted by a firstprinciple based Boltzmann transport equation calculation as a function of phonon-boundary scattering ${ }^{42}$. This comparison suggests $L_{a b}$ decreases from $>1 \mu \mathrm{m}$ to $\approx 200 \mathrm{~nm}$ for $x=0-0.34$.

Similarly, the comparison between our measured throughplane thermal conductivity of bulk $\mathrm{Li}_{x} \mathrm{MoS}_{2}$ and that predicted by first-principle based Boltzmann transport equation calculations suggests $L_{c} \approx 200 \mathrm{~nm}$ at $x=0$ (ref. 43). The lowest through-plane thermal conductivity in our bulk samples is comparable to that of turbostratic nano-crystalline $\mathrm{MoS}_{2}$ thin films deposited by magnetron sputtering ${ }^{36}$. In the turbostratic thin-film samples, the length of coherent stacking is unknown but should be less than the grain size $\approx 5 \mathrm{~nm}$ (ref. 44 ). The similar stacking disorder in our bulk samples $\mathrm{Li}_{x} \mathrm{MoS}_{2}$ suggests the $L_{c}<5 \mathrm{~nm}$ at $x=0.34$. A plausible explanation for this pronounced phonon mean-free path difference is that the lithium ions diffuse more easily along the in-plane direction compared with the through-plane direction. As a result, the density of disorder caused by lithium ion intercalation is less pronounced along the in-plane direction, Both $L_{c}$ and $L_{a b}$ reach their minimum values with the maximum mixing of the two phases at $x=0.34$.

Finally, we noticed that the through-plane thermal conductivity of thin-film $\mathrm{Li}_{x} \mathrm{MoS}_{2}$ sample is much lower than the in-plane thermal conductivity of the bulk $\mathrm{Li}_{x} \mathrm{MoS}_{2}$ sample, and the trends in through-plane thermal conductivity as a function of $x$ are different for bulk and thin-film samples. Compared with bulk $\mathrm{MoS}_{2}$, the basal planes in $\mathrm{Li}_{x} \mathrm{MoS}_{2}$ thin films contain high density of defects, especially point defects and oxygen impurities introduced during the chemical-vapour growth process of the thin sample. Defects can significantly reduce the thermal conductivity of $2 \mathrm{D}$ materials by enhancing phonon scattering ${ }^{45-47}$. Although the semiconductor $2 \mathrm{H}$ to metallic $1 \mathrm{~T}$ phase transition also occurs in the thin-film sample as indicated by the plateau in the discharge curve, unlike the bulk sample, the thermal conductivity of defective thin-film $\mathrm{Li}_{x} \mathrm{MoS}_{2}$ is more sensitive to lattice softening than stacking disorder ${ }^{47}$. On the basis of our elastic constants measurement results and estimation by LS equation, we attribute the decreasing trend of thermal conductivity in $\mathrm{Li}_{x} \mathrm{MoS}_{2}$ thin films with increasing lithium content $x$ to the softening of the lattice and the increased phonon-boundary scattering at the grain or phase boundaries ${ }^{48}$.

We investigated the anisotropic thermal transport in bulk crystal and CVD-grown thin-film $\mathrm{MoS}_{2}$ samples with different amounts of lithium ion intercalation. We demonstrated that intercalation impacts thermal transport in bulk and thin-film samples differently, depending on the crystalline quality of the $2 \mathrm{D}$ structure. In addition, we found that lithiation tends to reduce the phonon mean-free path more along the through-plane direction in $\mathrm{Li}_{x} \mathrm{MoS}_{2}$, rather than the in-plane direction, until the largest degree of disorder is reached at $x \approx 0.34$, leading to a significant increase in the thermal anisotropy ratio. This work provides insight on the impact of structural and compositional changes (for example, disorder, layer spacing and interfaces) on 2D materials applications, where thermal management is crucial.

\section{Methods}

Synthesis of thin-film $\mathbf{M o S}_{\mathbf{2}} \cdot \mathrm{MoS}_{2}$ thin films were grown on sapphire in a horizontal quartz tube furnace (Lindberg/Blue $\mathrm{M} 1,100^{\circ} \mathrm{C}$ Tube Furnace with 1 in diameter tube). The substrate was pre-deposited with $70 \mathrm{~nm}$ Mo thin film, which shows electrical conductivity of about $4.8 \times 10^{5} \mathrm{~S} \mathrm{~cm}^{-1}$. The Mo film was then placed in the centre of the tube furnace and S powder (Sigma Aldrich) was loaded in a ceramic crucible on the upstream cooler zone of the tube furnace. The temperature of the $\mathrm{S}$ was kept between 200 and $250^{\circ} \mathrm{C}$ when the centre of the tube furnace reaches $750{ }^{\circ} \mathrm{C}$. The tube was initially purged with $\mathrm{Ar}$ gas $(100 \mathrm{sccm})$ for 10 min to remove oxygen. During the chemical-vapour growth of $\mathrm{MoS}_{2}$ thin film, Ar gas flow rate was kept at $100 \mathrm{sccm}$, and the temperature of the tube furnace was quickly increased to $750^{\circ} \mathrm{C}$ in $15 \mathrm{~min}$ and then held for $20 \mathrm{~min}$, followed by natural cooling down.

Electrochemical intercalation of lithium. The thin-film and bulk $\mathrm{MoS}_{2}$ samples were assembled into battery configuration inside an argon-filled glovebox for lithium ion intercalation. Lithiation of thin-film $\mathrm{MoS}_{2}$ was performed in a glass vial. The thin-film $\mathrm{MoS}_{2}$ and a piece of lithium foil were used as the cathode and anode, respectively, and 1.0 M LiPF6 in 30:70 (vol\%) ethylene carbonate/dimethyl carbonate (Sol-Rite) as the electrolyte. The thin-film sample was wrapped around by a piece of stainless steel foil, which was used as the electrical contact. The discharge current was $14 \mu \mathrm{A}$. The lithium ion intercalation of the bulk $\mathrm{MoS}_{2}$ was carried out using 2025 coin cells, with $\mathrm{MoS}_{2}$ as the cathode, lithium foil as the anode, 1.0 M LiPF6 in 30:70 (vol\%) ethylene carbonate/dimethyl carbonate as electrolyte, and a Celgard 2400 separator. The discharge current used for the bulk $\mathrm{MoS}_{2}$ was $10 \mu \mathrm{A}$. After the discharge process, all samples were relaxed for days before they were cleaned by diethyl carbonate (anhydrous, Sigma Aldrich) inside the glovebox to remove the electrolyte left on the sample surfaces. Samples were sealed in air-tight aluminium pouches before they were transferred out of the glovebox and mailed from Ann Arbor, MI to Urbana, IL for sputtering deposition of $\mathrm{Al}$ or $\mathrm{NbV}$ for TDTR measurements.

TDTR measurements. TDTR was used to measure the thermal conductivity of bulk and thin-film intercalated $\mathrm{MoS}_{2}$. The experimental setup and model details on TDTR can be found elsewhere ${ }^{49,50}$. All the other details are shown in Supplementary Methods.

Before the TDTR measurements, metal thin films ( $\mathrm{Al}$ or $\mathrm{NbV}$ ) were deposited on the samples by magnetron sputtering. Samples were exposed to air for only $3-5 \mathrm{~s}$ before the process chamber was pumped down. We measured through-plane thermal conductivity of $\mathrm{MoS}_{2}$ at $f=9.8 \mathrm{MHz}$, with a $1 / e^{2}$ radius of the focused laser beams $w_{0}=11.7 \mu \mathrm{m}$. The thermal conductivity of $\mathrm{MoS}_{2}$ thin films reported in this work is the apparent (or effective) thermal conductivity of the thin film, including the two interfacial thermal resistances between $\mathrm{MoS}_{2}$ and the neighbouring materials, besides the intrinsic thermal resistance of the film. The sensitivity of the TDTR data to the in-plane thermal conductivity of $\mathrm{Li}_{x} \mathrm{MoS}_{2}$ thin film is small, which makes this in-plane thermal conductivity challenging to measure.

We measured through-plane thermal conductivity of bulk $\mathrm{MoS}_{2}$ at $f=9.8 \mathrm{MHz}$ with $w_{0}=11.7 \mu \mathrm{m}$. The thermal conductivity of bulk $\mathrm{MoS}_{2}$ and the interfacial thermal conductance between $\mathrm{Al}$ and $\mathrm{MoS}_{2}$ were fitted. The in-plane thermal conductivity of bulk $\mathrm{MoS}_{2}$ was measured using the beam-offset TDTR method as detailed in ref. 6 , at $f=1.1 \mathrm{MHz}$ with $w_{0} \approx 27 \mu \mathrm{m}$. NbV transducer was used in this measurement, whose thermal properties were characterized in ref. 51 . The total uncertainties of the measured thermal conductivity are calculated by taking into account the systematic errors that propagate from uncertainties in the film thickness, laser spot size and thermal properties of the transducer film and substrate. We have tried to use a $65 \mathrm{~nm}$-thick $\mathrm{NbV}$ thin film as the metal transducer to measure in-plane thermal conductivity $\Lambda$ of $\mathrm{MoS}_{2}$ thin film by TDTR method. However, due to the relatively low thermal conductance of the film, that is, $\Lambda d$, where $d$ is the thickness of the thin film the in-plane heat flow in metal transducer and the Sapphire substrate instead of the $\mathrm{MoS}_{2}$ thin film dominates the lateral heat flow which lead to a low sensitivity to the thin film in-plane thermal conductivity in TDTR measurement. The thermal conductivity measurements are performed at different locations on our samples to confirm the homogeneous distribution of lithium.

Elastic constants measurement. The elastic constants of the $\mathrm{Li}_{x} \mathrm{MoS}_{2}$ thin films were measured using pump-probe techniques. The polycrystalline $\mathrm{MoS}_{2}$ thin film with vertically aligned basal planes is transverse isotropic, which has five effective independent averaged elastic constants: $C_{11}^{\prime}, C_{12}^{\prime}, C_{13}^{\prime}, C_{33}^{\prime}$ and $C_{44}^{\prime}$. The error bars, $\sim 20 \%$, are calculated by taking into account the experimental errors and the systematic errors that propagate from uncertainties in the $\mathrm{Al}$ film thickness, $\mathrm{Li}_{x} \mathrm{MoS}_{2}$ film density and the input elastic constants.

Similarly, the elastic constants of bulk $\mathrm{Li}_{x} \mathrm{MoS}_{2}\left(C_{33}\right)$ were calculated from $\rho v_{L}^{2}$, where $\rho$ is calculated based on literature values of bulk $\mathrm{MoS}_{2}$ samples $\left(5.06 \mathrm{~g} \mathrm{~cm}^{-3}\right)$ and the degree of lithiation $x, \rho=5.06(160+7 x) / 160$. We deposited $\approx 10 \mathrm{~nm} \mathrm{NbV}$ on the bulk $\mathrm{Li}_{x} \mathrm{MoS}_{2}$ samples and used picosecond interferometry ${ }^{52}$ to determine the longitudinal speed of sound $v_{L}$. In the picosecond interferometry, the Brillouin scattering frequency $f_{\mathrm{B}}$ is related to the longitudinal speed of sound $v$ by $f_{\mathrm{B}}=2 n v_{L} / \lambda$, where $n$ is the index of refraction of the sample and $\lambda$ is the laser wavelength. We used the literature value of $n \approx 4.7$ at $\lambda=785 \mathrm{~nm}$ in this calculation $^{53}$. This measurement of picosecond interferometry uses the same experiment setup as TDTR. All the other details are shown in Supplementary Methods. 
Raman spectroscopy measurement. The Raman spectroscopy measurements were performed using an Acton Insight spectrometer (Princeton Instruments). The excitation wavelength is $488 \mathrm{~nm}$ from a Spectra-Physics Cyan (CDRH) solid-state laser. A power of $\approx 1 \mathrm{~mW}$ is used to avoid excessive sample heating. We used an optical configuration similar to that d multilayer graphene $e^{54}$; the laser plasma lines are removed using a BragGrate bandpass filter (OptiGrate), while the Rayleigh line is suppressed using three BragGrate notch filters (OptiGrate) in series each with an optical density 3 and a spectral bandwidth $5-10 \mathrm{~cm}^{-1}$. The backscattered signal was collected through a $20 \times$ objective (numerical aperture $=0.4$ ) with laser spot size $\approx 10 \mu \mathrm{m}$ at the sample surface and dispersed by a $1,200 \mathrm{~g} \mathrm{~mm}^{-1}$ grating with a spectral resolution $\approx 2 \mathrm{~cm}^{-1}$.

Additional structural analysis. TEM specimens of the thin-film $\mathrm{MoS}_{2}$ samples were prepared using a FEI FIB200 Focused Ion Beam system. The bulk TEM specimens were prepared by hand grinding and ultrasonication in ethanol. TEM was performed using a Hitachi HD 2300 STEM. X-ray diffraction was performed using a Rigaku SmartLab x-ray diffractometer.

Data availability. The data supporting the main findings of this study are available from the corresponding authors on request.

\section{References}

1. Wang, Q. H., Kalantar-Zadeh, K., Kis, A., Coleman, J. N. \& Strano, M. S. Electronics and optoelectronics of two-dimensional transition metal dichalcogenides. Nat. Nanotechnol. 7, 699-712 (2012).

2. Wang, H. et al. Integrated circuits based on bilayer $\mathrm{MoS}_{2}$ transistors. Nano. Lett. 12, 4674-4680 (2012).

3. Zeng, H., Dai, J., Yao, W., Xiao, D. \& Cui, X. Valley polarization in $\mathrm{MoS}_{2}$ monolayers by optical pumping. Nat. Nanotechnol. 7, 490-493 (2012).

4. Cheng, R. et al. Few-layer molybdenum disulfide transistors and circuits for high-speed flexible electronics. Nat. Commun. 5, 5143 (2014).

5. Wan, C. et al. Flexible n-type thermoelectric materials by organic intercalation of layered transition metal dichalcogenide $\mathrm{TiS}_{2}$. Nat. Mater. 14, 622-627 (2015).

6. Liu, J., Choi, G.-M. \& Cahill, D. G. Measurement of the anisotropic thermal conductivity of molybdenum disulfide by the time-resolved magneto-optic Kerr effect. J. Appl. Phys. 116, 233107 (2014).

7. Jo, I., Pettes, M. T., Ou, E., Wu, W. \& Shi, L. Basal-plane thermal conductivity of few-layer molybdenum disulfide. Appl. Phys. Lett. 104, 201902 (2014).

8. Yan, R. et al. Thermal conductivity of monolayer molybdenum disulfide obtained from temperature-dependent raman spectroscopy. ACS Nano 8, 986-993 (2014).

9. Xiong, F. et al. Li intercalation in $\mathrm{MoS}_{2}$ : in situ observation of its dynamics and tuning optical and electrical properties. Nano. Lett. 15, 6777-6784 (2015).

10. Py, M. A. \& Haering, R. R. Structural destabilization induced by lithium intercalation in $\mathrm{MoS}_{2}$ and related compounds. Can. J. Phys. 61, 76-84 (1983).

11. Imanishi, N., Toyoda, M., Takeda, Y. \& Yamamoto, O. Study on lithium intercalation into $\mathrm{MoS}_{2}$. Solid State Ionics 58, 333-338 (1992).

12. Yoo, E. et al. Large reversible Li storage of graphene nanosheet families for use in rechargeable lithium ion batteries. Nano. Lett. 8, 2277-2282 (2008).

13. Luckyanova, M. N. et al. Anisotropy of the thermal conductivity in GaAs/AlAs superlattices. Nano. Lett. 13, 3973-3977 (2013).

14. Renteria, J. D. et al. Strongly anisotropic thermal conductivity of free-standing reduced graphene oxide films annealed at high temperature. Adv. Funct. Mater. 25, 4664-4672 (2015).

15. Kong, D. et al. Synthesis of $\mathrm{MoS}_{2}$ and $\mathrm{MoSe}_{2}$ films with vertically aligned layers. Nano. Lett. 13, 1341-1347 (2013).

16. Chen, J. M. \& Wang, C. S. Second order Raman spectrum of $\mathrm{MoS}_{2}$. Solid State Commun. 14, 857-860 (1974).

17. Zhang, X. et al. Phonon and Raman scattering of two-dimensional transition metal dichalcogenides from monolayer, multilayer to bulk material. Chem. Soc. Rev. 44, 2757-2785 (2015).

18. Zhang, R., Upreti, S. \& Stanley Whittingham, M. Tin-iron based nano-materials as anodes for Li-Ion batteries. J. Electrochem. Soc. 158, A1498-A1504 (2011).

19. Stephenson, T., Li, Z., Olsen, B. \& Mitlin, D. Lithium ion battery applications of molybdenum disulfide $\left(\mathrm{MoS}_{2}\right)$ nanocomposites. Energy Environ. Sci. 7, 209-231 (2014)

20. Cahill, D. G., Watson, S. K. \& Pohl, R. O. Lower limit to the thermal conductivity of disordered crystals. Phys. Rev. B 46, 6131-6140 (1992)

21. Cahill, D. G., Melville, A., Schlom, D. G. \& Zurbuchen, M. A. Low thermal conductivity of $\mathrm{CsBiNb}_{2} \mathrm{O}_{7}$ epitaxial layers. Appl. Phys. Lett. 96, 121903 (2010)

22. Li, D., Zhao, P., Zhao, J.-C. \& Cahill, D. G. Generation and detection of gigahertz surface acoustic waves using an elastomeric phase-shift mask. J. Appl. Phys. 114, 143102 (2013).

23. Taylor, B., Maris, H. J. \& Elbaum, C. Phonon focusing in solids. Phys. Rev. Lett. 23, 416-419 (1969).
24. Xiao, J. et al. Electrochemically induced high capacity displacement reaction of $\mathrm{PEO} / \mathrm{MoS}_{2}$ /graphene nanocomposites with lithium. Adv. Funct. Mater. 21, 2840-2846 (2011).

25. Shannon, R. Revised effective ionic radii and systematic studies of interatomic distances in halides and chalcogenides. Acta Crystallogr. A 32, 751-767 (1976).

26. Azhagurajan, M., Kajita, T., Itoh, T., Kim, Y.-G. \& Itaya, K. In situ visualization of lithium ion intercalation into $\mathrm{MoS}_{2}$ single crystals using differential optical microscopy with atomic layer resolution. J. Am. Chem. Soc. 138, 3355-3361 (2016).

27. Wang, L., Xu, Z., Wang, W. \& Bai, X. Atomic mechanism of dynamic electrochemical lithiation processes of $\mathrm{MoS}_{2}$ nanosheets. J. Am. Chem. Soc. 136, 6693-6697 (2014).

28. Wypych, F. \& Schollhorn, R. 1T- $\mathrm{MoS}_{2}$, a new metallic modification of molybdenum disulfide. J. Chem. Soc. Chem. Commun. 19, 1386-1388 (1992).

29. Julien, C., Sekine, T. \& Balkanski, M. Lattice dynamics of lithium intercalated $\mathrm{MoS}_{2}$. Solid State Ionics 48, 225-229 (1991).

30. Dungey, K. E., Curtis, M. D. \& Penner-Hahn, J. E. Structural characterization and thermal stability of $\mathrm{MoS}_{2}$ intercalation compounds. Chem. Mater. 10, 2152-2161 (1998).

31. Lukowski, M. A. et al. Enhanced hydrogen evolution catalysis from chemically exfoliated metallic $\mathrm{MoS}_{2}$ nanosheets. J. Am. Chem. Soc. 135, 10274-10277 (2013).

32. Ahmed, T., Modine, N. A. \& Zhu, J.-X. Bonding between graphene and $\mathrm{MoS}_{2}$ monolayers without and with Li intercalation. Appl. Phys. Lett. 107, 043903 (2015).

33. Julien, C. M. Lithium intercalated compounds: charge transfer and related properties. Mater. Sci. Engineer. Rep. 40, 47-102 (2003).

34. Rocquefelte, X. et al. Mo cluster formation in the intercalation compound $\mathrm{LiMoS}_{2}$. Phys. Rev. B 62, 2397-2400 (2000).

35. Xiong, F. et al. Li intercalation in $\mathrm{MoS}_{2}$ : in situ observation of its dynamics and tuning optical and electrical properties. Nano. Lett. 15, 6777-6784 (2015).

36. Muratore, C. et al. Cross-plane thermal properties of transition metal dichalcogenides. Appl. Phys. Lett. 102, 081604 (2013).

37. Chiritescu, C. et al. Ultralow thermal conductivity in disordered, layered $\mathrm{WSe}_{2}$ crystals. Science 315, 351-353 (2007).

38. Mavrokefalos, A., Nguyen, N. T., Pettes, M. T., Johnson, D. C. \& Shi, L. In-plane thermal conductivity of disordered layered $\mathrm{WSe}_{2}$ and $(\mathrm{W}) \mathrm{x}\left(\mathrm{WSe}_{2}\right) \mathrm{y}$ superlattice films. Appl. Phys. Lett. 91, 171912 (2007).

39. McCurdy, A. K., Maris, H. J. \& Elbaum, C. Anisotropic heat conduction in cubic crystals in the boundary scattering regime. Phys. Rev. B 2, 4077-4083 (1970).

40. Minnich, A. J. Thermal phonon boundary scattering in anisotropic thin films. Appl. Phys. Lett. 107, 183106 (2015).

41. Zheng, Q., Braun, P. V. \& Cahill, D. G. Thermal conductivity of graphite thin films grown by low temperature chemical vapor deposition on $\mathrm{Ni}$ (111). Adv. Mater. Interfaces 3, 1600234 (2016).

42. Gu, X. \& Yang, R. Phonon transport in single-layer transition metal dichalcogenides: a first-principles study. Appl. Phys. Lett. 105, 131903 (2014).

43. Gu, X., Li, B. \& Yang, R. Layer thickness-dependent phonon properties and thermal conductivity of $\mathrm{MoS}_{2}$. J. Appl. Phys. 119, 085106 (2016).

44. Muratore, C. et al. Thermal anisotropy in nano-crystalline $\mathrm{MoS}_{2}$ thin films. Phys. Chem. Chem. Phys. 16, 1008-1014 (2014).

45. Ding, Z., Pei, Q.-X., Jiang, J.-W. \& Zhang, Y.-W. Manipulating the thermal conductivity of monolayer $\mathrm{MoS}_{2}$ via lattice defect and strain engineering. J. Phys. Chem. C 119, 16358-16365 (2015).

46. Pop, E., Varshney, V. \& Roy, A. K. Thermal properties of graphene: fundamentals and applications. MRS Bull. 37, 1273-1281 (2012).

47. Erhart, P., Hyldgaard, P. \& Lindroth, D. O. Microscopic origin of thermal conductivity reduction in disordered van der Waals solids. Chem. Mater. 27, 5511-5518 (2015).

48. Guilmeau, E., Maignan, A., Wan, C. \& Koumoto, K. On the effects of substitution, intercalation, non-stoichiometry and block layer concept in $\mathrm{TiS}_{2}$ based thermoelectrics. Phys. Chem. Chem. Phys. 17, 24541-24555 (2015).

49. Liu, J. et al. Simultaneous measurement of thermal conductivity and heat capacity of bulk and thin film materials using frequency-dependent transient thermoreflectance method. Rev. Sci. Instrum. 84, 034902 (2013).

50. Cahill, D. G. Analysis of heat flow in layered structures for time-domain thermoreflectance. Rev. Sci. Instrum. 75, 5119-5122 (2004)

51. Feser, J. P., Liu, J. \& Cahill, D. G. Pump-probe measurements of the thermal conductivity tensor for materials lacking in-plane symmetry. Rev. Sci. Instrum. 85, 104903 (2014).

52. O’Hara, K. E., Hu, X. \& Cahill, D. G. Characterization of nanostructured metal films by picosecond acoustics and interferometry. J. Appl. Phys. 90, 4852-4858 (2001).

53. Beal, A. R. \& Hughes, H. P. Kramers-Kronig analysis of the reflectivity spectra of $2 \mathrm{H}-\mathrm{MoS}_{2}, 2 \mathrm{H}-\mathrm{MoSe}_{2}$ and $2 \mathrm{H}-\mathrm{MoTe}_{2}$. J. Phys. C . 12, 881 (1979). 
54. Tan, P. H. et al. The shear mode of multilayer graphene. Nat. Mater. 11, 294-300 (2012).

55. Wang, H. et al. $\mathrm{MoSe}_{2}$ and WSe $\mathrm{W}_{2}$ Nanofilms with vertically aligned molecular layers on curved and rough surfaces. Nano. Lett. 13, 3426-3433 (2013).

\section{Acknowledgements}

We thank the analysis lab in School of Chemical Sciences in University of Illinois at Urbana-Champaign for assistance with ICP-MS analysis. TDTR and RBS measurements were done using the equipment in the Frederick Seitz Materials Research Laboratory at University of Illinois at Urbana-Champaign.

\section{Author contributions}

G.Z., J.L. and D.G.C. conceived the samples and measurements. G.Z. and R.Z. performed electrochemical experiments and sample fabrications. J.L. performed measurements of thermal properties, elastic properties, RBS, and ICP-MS. G.Z., J.L. and Q.Z. performed structural analysis. Q.Z. carried out the Raman Spectroscopy measurements. J.L. and D.L. analysed the shear modulus. G.Z., J.L. and Q.Z. analysed data, designed figures and wrote the manuscript with comments from all co-authors. D.G.C. and D.B. coordinated the project.

\section{Additional information}

Supplementary Information accompanies this paper at http://www.nature.com/ naturecommunications

Competing financial interests: The authors declare no competing financial interests.

Reprints and permission information is available online at http://npg.nature.com/ reprintsandpermissions/

How to cite this article: Zhu, G. et al. Tuning thermal conductivity in molybdenum disulfide by electrochemical intercalation. Nat. Commun. 7, 13211 doi: 10.1038/ ncomms13211 (2016). (c) (i) International License. The images or other third party material in this article are included in the article's Creative Commons license, unless indicated otherwise in the credit line; if the material is not included under the Creative Commons license, users will need to obtain permission from the license holder to reproduce the material. To view a copy of this license, visit http://creativecommons.org/licenses/by/4.0/ 